\title{
Negative effects of item repetition on source memory
}

\author{
Kyungmi Kim • Do-Joon Yi • Carol L. Raye • \\ Marcia K. Johnson
}

Published online: 13 March 2012

(C) Psychonomic Society, Inc. 2012

\begin{abstract}
In the present study, we explored how item repetition affects source memory for new item-feature associations (picture-location or picture-color). We presented line drawings varying numbers of times in Phase 1. In Phase 2, each drawing was presented once with a critical new feature. In Phase 3, we tested memory for the new source feature of each item from Phase 2. Experiments 1 and 2 demonstrated and replicated the negative effects of item repetition on incidental source memory. Prior item repetition also had a negative effect on source memory when different source dimensions were used in Phases 1 and 2 (Experiment 3) and when participants were explicitly instructed to learn source information in Phase 2 (Experiments 4 and 5). Importantly, when the order between Phases 1 and 2 was reversed, such that item repetition occurred after the encoding of critical itemsource combinations, item repetition no longer affected source memory (Experiment 6). Overall, our findings did not support predictions based on item predifferentiation, within-dimension source interference, or general interference from multiple traces of an item. Rather, the findings were consistent with the idea that prior item repetition reduces attention to subsequent presentations of the item, decreasing the likelihood that critical item-source associations will be encoded.
\end{abstract}

\footnotetext{
K. Kim $(\bowtie) \cdot$ C. L. Raye $\cdot$ M. K. Johnson

Department of Psychology, Yale University,

2 Hillhouse Avenue,

New Haven, CT 06520-8205, USA

e-mail: kyungmi.kim@yale.edu

D.-J. Yi

Department of Psychology, Yonsei University, Seoul, Korea
}

Keywords Item repetition · Source memory · Novelty · Reminding

Much of daily life consists of events involving familiar people, objects, and places. Thus, understanding how prior experiences affect the formation of new memories involving familiar things is fundamental for advancing our understanding of how memory functions under everyday circumstances. Episodic remembering requires more than the recognition or recall of items (e.g., words or pictures, as defined by an experimenter; Chalfonte \& Johnson, 1996). It involves the recognition or recall of items along with other source features (e.g., time, place, color, emotion experienced, or cognitive operations engaged) associated with each item (Gardiner \& Richardson-Klavehn, 2000; Johnson, Hashtroudi, \& Lindsay, 1993). That is, episodic remembering involves the subjective experience of cohesive events consisting of multiple features that are bound together (Johnson \& Raye, 1981; Tulving, 1983). Repeated presentation of items typically improves recognition or recall of them (Hintzman, 1976). Similarly, memory for source information (e.g., list membership) typically benefits from repeated presentations of an item-source combination (e.g., intralist item repetition during study; Hintzman \& Waters, 1970; Newby, 1981, Exp. 2). However, little is known about how prior encounters with an item affect memory for new source information from subsequent encounters with the same item. Is subsequent source memory affected by prior experiences with an item (i.e., item repetition)? If so, what are the mechanisms underlying the effects of prior item repetition on source memory?

Various theoretical ideas and empirical findings provide different rationales for predicting how prior item repetition might affect source memory. One possibility, extending the 
idea of stimulus learning/predifferentiation (e.g., Gibson, 1940, 1953), is that prior experiences with an item will facilitate source memory by reducing the attentional resources necessary for item processing during the binding of new item-source information. A large body of literature has shown that prior experience with a stimulus facilitates subsequent processing of that same item - for example, producing faster and more accurate perceptual identification or stimulus classification (behavioral priming; Roediger \& McDermott, 1993; Tulving \& Schacter, 1990). Support for the idea that repetition facilitates item processing has also come from studies using words that vary in normative frequency. In a dual-task paradigm, performance on a secondary task shows faster response times and greater accuracy during encoding of high-frequency words relative to low-frequency words (Naveh-Benjamin, Craik, Guez, \& Dori, 1998; Naveh-Benjamin \& Guez, 2000). Using a picture-word interference paradigm, Miozzo and Caramazza (2003) found that when participants were presented with pictures and asked to name each picture as quickly as possible, naming latencies were faster when the pictures were presented along with high-frequency words than when they were presented with low-frequency words. Importantly, this difference in naming latencies for pictures accompanied by high- versus low-frequency words was reduced when participants read aloud the words several times prior to the picture-naming task. In a similar paradigm, Diana and Reder (2006, Exp. 1) further demonstrated that pairing a high-frequency word with a picture led to better memory for the picture than did pairing it with a low-frequency word. Extending these findings to source memory, such repetition benefits for processing items should improve item-source feature binding. To the extent that limited attention is available to encode events, the processing advantage for an item that has been repeatedly presented before should leave more attention available for processing source information (e.g., location or color).

Alternatively, prior item occurrences could impair source memory. This would be expected on the basis of two different lines of argument:

\section{(a) Greater interference from source information associated with more frequently presented items}

Frequently presented items may acquire source information that competes with the retrieval of target source features (proactive or retroactive interference; Postman, 1971; Underwood, 1949). According to a multiple-trace account of memory (e.g., Minerva 2; Hintzman, 1988), each time that an item is presented, it forms a memory trace that contains both the item and its source information. For item recognition memory, all traces involving that item can contribute to the judgment of an item as "old." In contrast, source memory requires the revival of a particular trace relevant to the current goals/demands, which is affected by interference from irrelevant memory traces. Thus, reviving any specific trace may suffer from interference as a function of the number of times that the item was previously encountered. For instance, item recall and source memory are better for words with low rather than high preexperimental context variability (i.e., that are associated with only a few vs. many different everyday contexts; Hicks, Marsh, \& Cook, 2005; Marsh, Cook, \& Hicks, 2006). In a study in which item frequency was manipulated, A-B word pairs were initially presented, followed by zero, one, or two presentations of B words alone. At test, the probability of recalling an A word in response to the corresponding $\mathrm{B}$ word decreased as a function of the number of times that the B word had been presented alone, suggesting that memory traces formed when $\mathrm{B}$ words were presented alone interfered with recalling the trace of an initial A-B presentation (Overton \& Adolphson, 1979, Exp. 2).

(b) Less efficacious encoding of item-source feature associations for items previously presented more frequently

This prediction can be derived by extending the novelty-encoding hypothesis (Tulving \& Kroll, 1995), attention-likelihood theory (Glanzer \& Adams, 1990), or the recursive reminding hypothesis (Hintzman, 2004) from item memory to source memory. While they vary in the specific mechanisms suggested, each of these theories can be construed to predict that prior experiences with an item will reduce the probability that source information during a particular episode will be bound to the item at encoding.

When participants are repeatedly preexposed to some items but not others prior to the study phase of a critical list, recognition memory for the critical list items is typically superior for items that were not presented prior to the study phase ("novel" items) relative to familiar ones (Äberg \& Nilsson, 2001; Kormi-Nouri, Nilsson, \& Ohta, 2005; Tulving \& Kroll, 1995; but see Poppenk, Köhler, \& Moscovitch, 2010). According to the noveltyencoding hypothesis, this novelty effect occurs because the efficacy of encoding online information is determined by the novelty of that information. It is assumed that a specific novelty detection brain network involving a hippocampal "novelty signal" supports this selective encoding advantage of novel information at an early phase of encoding, which screens out familiar items from later, higher-level processing (Lisman \& Grace, 2005; Tulving, Markowitsch, Craik, Habib, \& Houle, 1996). Support for this hypothesis has come from neuroimaging studies showing greater medial temporal lobe (MTL) activity for novel than for repeated stimuli (Kumaran \& Maguire, 2006; Tulving et al., 1996) and overlapping hippocampal regions for areas activated during the encoding of novel items and those associated with subsequent memory effects (Kirchhoff, Wagner, Maril, \& Stern, 
2000). If prioritized elaborative encoding of novel information incorporates not only the item but also its associated source features (e.g., as evidenced by the greater probability of "remember" as well as "know" judgments for novel than for nonnovel items: Kishiyama \& Yonelinas, 2003), then source memory should be poorer for items with relatively more prior presentations.

Attention-likelihood theory would make a similar prediction. Originally proposed to account for a lower hit rate and higher false alarm rate in recognition memory for highfrequency than for low-frequency words in the English language (the mirror effect; Glanzer \& Adams, 1985, 1990; Glanzer, Adams, Iverson, \& Kim, 1993), attentionlikelihood theory suggests that low-frequency items receive more attention than do high-frequency items at encoding, and thus have more features "marked" in the memory trace. Generalizing the idea of the features of an item to include other source features, the source features of low-frequency, as compared to higher-frequency, items would receive relatively more attention at encoding. This prediction has received support from studies showing more accurate source judgments for low-frequency words than for high-frequency words (Diana \& Reder, 2006, Exp. 3; Rugg, Cox, Doyle, \& Wells, 1995), as well as a lowfrequency advantage for recollection and a smaller advantage for familiarity (Guttentag \& Carroll, 1997).

Finally, a prediction can be derived from the recursive reminding hypothesis (Hintzman, 2004, 2010). The recursive reminding hypothesis posits that when an item is presented for a second time, it reminds one of the item's first presentation (i.e., spontaneous reactivation). Consistent with the idea that memories include information about the cognitive operations active during encoding (Johnson et al., 1993), the subjective experience of being reminded of an item's first presentation is encoded in the memory trace of the second presentation. Thus, when an item is repeatedly experienced, iterative reminding and encoding across each presentation yields recursive representations in which early remindings are embedded in later remindings - information that, for example, could underlie judgments of frequency. Of critical importance for the present study, remindings are proposed to direct attention to internal representations and away from perceptual stimuli (Hintzman, 2004). The recursive reminding hypothesis, as applied here, is consistent with the idea of a trade-off relationship between internally directed, reflective attention and externally directed, perceptual attention to external stimuli (e.g., Chun \& Johnson, 2011), which should result in reduced encoding of source information for more frequently presented items.

To investigate the effects of item repetition on source memory accuracy, in the present experiments we used a three-phase procedure. In Phase 1 , we presented items a varying number of times. In Phase 2, each item was presented once with a specific source feature (location or color). In Phase 3, individuals' memory for the specific source in Phase 2 of each item was probed. In Experiments 1 and 2, we demonstrated and replicated a negative effect of prior item repetition on the accuracy of source memory. Given that this repetition effect was negative, we then explored whether greater source interference for more frequently presented items would occur when a new, salient feature dimension was introduced in Phase 2 (Exp. 3) and when participants intentionally directed attention to learning critical source information in Phase 2 (Exp. 4). In Experiment 5, to assess the impact of item novelty on source memory, we added a novel condition in which items only appeared in Phases 2 and 3 (i.e., no presentation in Phase 1). Finally, in Experiment 6, we reversed the order of Phases 1 and 2, to explore whether the locus of item repetition effects is at encoding or retrieval.

\section{Experiment 1}

In Experiment 1, we investigated the effects of varying prior presentation frequencies of pictures (Phase 1) on individuals' ability to later remember (Phase 3 ) each picture's location on the screen in Phase 2. In Phase 3, old items were intermixed with new items at test, and source memory accuracy was conditionalized on correct item recognition.

Method

\section{Participants}

A group of 21 undergraduate students (mean age: 19.7 [ \pm 2.45$]$; 12 female, 9 male) at Yale University participated for course credit (the participants were drawn from the same pool for all experiments). The data from one participant, who was aware of the surprise memory test in advance, were excluded from the analysis (thus, $N=20$ ).

\section{Design and materials}

The experiment had one within-subjects independent factor (item preexposure frequency: low, medium, or high). The stimulus set was 64 black-and-white line drawings of common objects (32 living and 32 nonliving; Snodgrass \& Vanderwart, 1980). Overall, 48 drawings served as critical items, and the remaining 16 served as "new" items for the old/new item recognition and source memory test. For each participant, eight living and eight nonliving items were randomly assigned to each of four conditions (low, medium, high, and new). 


\section{Procedure}

A schematic view of the procedure is shown in Fig. 1. The experiment consisted of three phases: item preexposure, itemsource feature association, and surprise item-recognition/ source memory test.

Phase 1. Item preexposure. On each trial, after a 200-ms fixation, one of 48 critical items was presented in the center of a gray background screen for $1 \mathrm{~s}$. Trials were separated by a $500-\mathrm{ms}$ intertrial interval (ITI). Participants' task was to decide for each picture whether it depicted a living or nonliving object (animacy judgment) by pressing one of two buttons corresponding to "living" and "nonliving," respectively. Each picture was presented one, four, or 16 time(s) to introduce low, medium, or high levels of item frequency, respectively, resulting in a total of 336 trials. The order of the trials was random.

Phase 2. Item-source feature association. Each Phase 2 trial began with a 200-ms fixation, followed by the presentation of a black-outlined square frame in each of the four quadrants of a computer screen. After $500 \mathrm{~ms}$, a critical item was presented inside one of the frames for $2 \mathrm{~s}$ with a 500-ms ITI separating trials. The 16 objects in each condition (low, medium, and high item frequency) were each presented once, randomly assigned to one of the four locations, with the restriction that four objects occurred in each location. Participants were asked to perform the same living/nonliving judgment as in Phase 1. There were 48 trials (i.e., critical items) presented in random order.
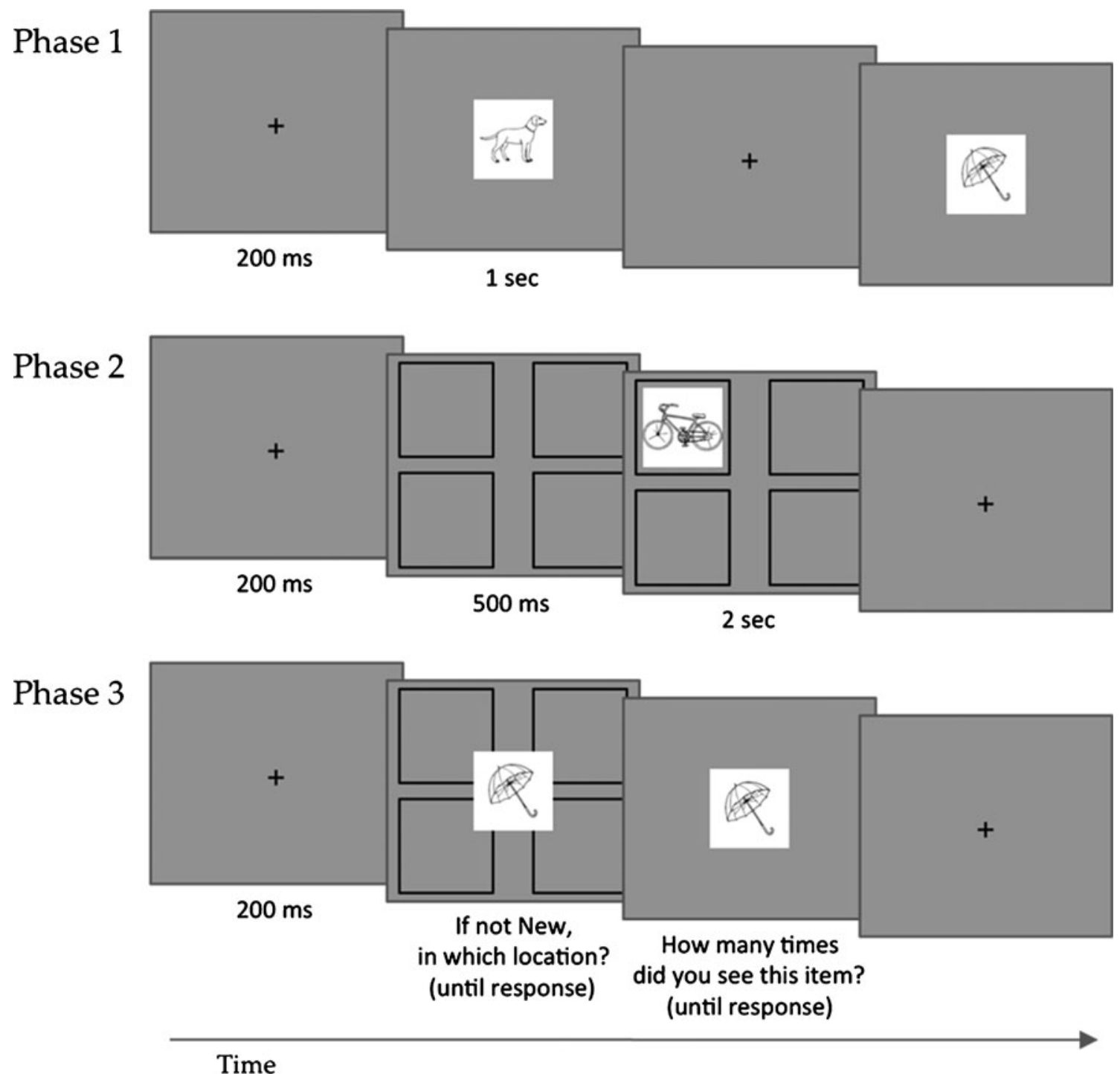

Fig. 1 Schematic view of the procedure (Exp. 1) 
Phase 3. Surprise item-recognition/source memory test. Immediately following Phase 2, participants took a surprise memory test. Each Phase 3 trial consisted of a 200-ms fixation, followed by a picture of an object in the center of the screen superimposed on four frames designating quadrants. All 48 old items (i.e., the critical items) were presented, along with 16 new items from the same stimulus set. For each object, participants were asked to indicate whether it was presented in Phase 2 in Quadrant 1, 2, 3, or 4, or whether it was new (not seen in the previous phases). The recognition/source judgment was followed by a frequency judgment task in which participants were asked to judge the overall presentation frequency of the item during the two preceding phases, on a scale ranging from 1 to 20 presentation(s). Both the memory and frequency judgment trials were self-paced.

Upon completion of the experimental phases, participants answered a brief postexperimental questionnaire that assessed their awareness of the experimental hypothesis and whether they expected a memory test: The participants were asked to describe any thoughts about the purpose and/or hypothesis of the study and to rate how much they had anticipated a memory test, on a scale ranging from 1 (not at all) to 5 (very much). In all experiments reported here, we excluded data from any participant who guessed the actual experimental hypothesis. For experiments involving incidental learning (all of the experiments except Exps. 4 and 5), we additionally excluded data from participants who gave a rating of 4 or 5 on the memory anticipation scale.

\section{Statistical analysis}

A one-way repeated measures analysis of variance (ANOVA) was used for each experiment reported in the present paper, with the within-subjects factor of Item Frequency (three levels in Exps. 1 through 4 and Exp. 6, and four levels in Exp. 5). Where applicable, a Greenhouse-Geisser correction was used to account for violation of the sphericity assumption.

Results and discussion

\section{Recognition memory}

The proportion of objects correctly recognized as "old" (i.e., the total number of old items minus the number of old items identified as "new" [misses], divided by the total number of old items) was computed for each item frequency condition and subjected to analysis. We found a significant effect of item frequency, $F(1.527,29.018)=$ $35.57, p<.001, \eta_{\mathrm{p}}{ }^{2}=.65$, with a linear trend, $F(1,19)=$ 43.58, $p<.001, \eta_{\mathrm{p}}{ }^{2}=.70$. High-frequency items $(98.75 \%)$ were recognized better than medium-frequency items $(92.19 \%)$, which in turn were recognized better than low-frequency items $(77.50 \%)$. The overall false alarm rate for distractors (i.e., the proportion of new items called "old") was $0.94 \%$.

\section{Source memory}

Source memory was calculated by dividing the number of correct location assignments by the total number of items that were correctly recognized as old for each of the item frequency conditions. The effect of item frequency was significant, $F(2,38)=20.93, p<.001, \eta_{\mathrm{p}}{ }^{2}=.52$ (Fig. 2a), with a linear trend, $F(1,19)=44.53, p<.001, \eta_{\mathrm{p}}{ }^{2}=.70$. Participants' memory for location was best in the lowfrequency condition $(56.47 \%)$, followed by the mediumfrequency condition (43.05\%), and then the highfrequency condition $(33.83 \%)$.

\section{Frequency judgment}

The effect of item frequency was significant, $F(2,38)=$ 94.57, $p<.001, \eta_{\mathrm{p}}{ }^{2}=.83$. The mean frequencies were $3.39,6.37$, and 12.26 for the low-, medium-, and highfrequency conditions, respectively. ${ }^{1}$ Given that each picture was presented one, four, or 16 times in Phase 1 and once in Phase 2, the correct frequencies for the low-, medium-, and high-frequency conditions were 2, 5, and 17, respectively. The overestimation of low-frequency items and the underestimation of high-frequency items is often found (e.g., Howell, 1973).

The findings of Experiment 1 demonstrated that memory for Phase 2 location was poorer as the number of Phase 1 item repetitions increased, whereas item recognition was better. Given that recognition can be based on item familiarity, memory for the episodic details of an item's occurrence, or both (Johnson et al., 1993; Kelley \& Jacoby, 2000), these results suggest that, relative to less-frequent items, more frequently presented items were less likely to be recognized on the basis of recollection of critical location information from Phase 2.

\footnotetext{
${ }^{1}$ In all of the experiments reported in this article, we observed the expected pattern of results with respect to the effect of item repetition on participants' estimates of item presentation frequency. For brevity, the frequency judgment data are not described in the Results sections of the subsequent experiments.
} 

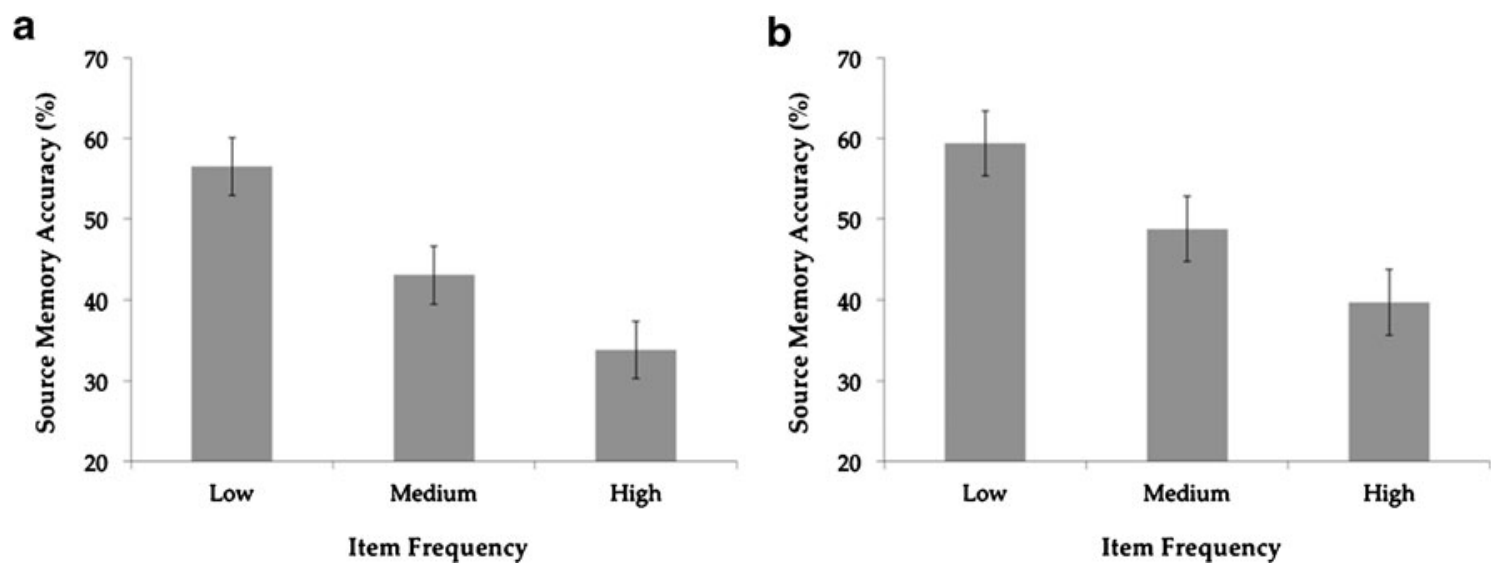

Fig. 2 Source memory accuracy as a function of item frequency in (a) Experiment 1 and (b) Experiment 2. Error bars represent 95\% withinsubjects confidence intervals (Loftus \& Masson, 1994)

\section{Experiment 2}

In Experiment 1, the familiarity of "old" items at test presumably differed according to whether the items had previously been seen one, four, or 16 times. It is possible that participants were reluctant to say "old" to items seen fewer times unless they also remembered the source information. Thus, relative to low-frequency items, for high-frequency items, source judgments might have been made for more items for which the source information was weaker, which in turn might have resulted in poorer source memory for the more frequently presented items. In Experiment 2, we attempted to reduce this possibility by using a forced choice source memory test involving only "old" items in Phase 3. Thus, unlike in Experiment 1, source judgments were required for all items previously seen.

Method

\section{Participants}

A group of 20 undergraduate students (mean age: 19.2 [ \pm 1.15$]$; 11 female, 9 male) participated for course credit.

\section{Design and materials}

The experimental design was the same as in Experiment 1. The stimuli consisted of 48 pictures (i.e., critical items) from the same stimulus set as in Experiment 1.

\section{Procedure}

The procedure for Phases 1 and 2 was the same as in Experiment 1. In Phase 3, only the critical items were presented, and participants indicated for each picture whether it had been presented in Quadrant 1, 2, 3, or 4 during
Phase 2. After the source memory judgment for each picture, participants were asked to indicate how confident they were about their memory for location on a 5-point scale $(1=$ the lowest and $5=$ the highest) by pressing a corresponding keyboard button. The frequency judgment followed the confidence rating. The trials were self-paced.

Results and discussion

\section{Source memory}

The source memory pattern was the same as in Experiment 1 (Fig. 2b). A significant effect of item frequency emerged, $F(2,38)=12.17, p<.001, \eta_{\mathrm{p}}{ }^{2}=.39$, which showed a linear trend, $F(1,19)=22.31, p<.001, \eta_{\mathrm{p}}{ }^{2}=.54$ : Participants' source memory performance was best in the low-frequency condition $(59.38 \%)$, followed by the medium-frequency condition $(48.75 \%)$, and then the high-frequency condition (39.69\%).

\section{Confidence ratings}

Confidence ratings also showed a significant effect of item frequency, $F(2,38)=17.26, p<.001, \eta_{\mathrm{p}}{ }^{2}=.46$. Exhibiting a linear trend, $F(1,19)=28.23, p<.001, \eta_{\mathrm{p}}{ }^{2}=.60$, the mean confidence scores were $3.59,3.11$, and 2.82 for the low-, medium-, and high-frequency conditions, respectively. ${ }^{2}$

Experiment 2 replicated the findings from Experiment 1: When participants were forced to make source judgments about all items, the accuracy of memory for Phase 2 location decreased as item frequency increased. Source memory

\footnotetext{
$\overline{2}$ In this and subsequent experiments, the confidence data largely show the same pattern as the accuracy data. Therefore, we will not discuss the confidence data further.
} 
accuracy was accompanied by a corresponding pattern of subjective confidence for source judgments. These findings are in line with previous studies showing higher confidence ratings for items given higher ratings on subjective source features (Henkel, Franklin, \& Johnson, 2000; Lyle \& Johnson, 2006) or for items given "remember" versus "know" judgments (Holmes, Waters, \& Rajaram, 1998; Wixted \& Stretch, 2004). Overall, the findings from Experiments 1 and 2 did not support the predifferentiation hypothesis that better source memory would result from facilitated processing in Phase 2 of previously experienced items.

\section{Experiment 3}

It is possible that in Experiments 1 and 2, items acquired location information in Phase 1 (when all items appeared in the center), which might have interfered with retrieval of the location information presented in Phase 2. If so, an item's association with the center location should have been greater, the more frequently the item was presented in Phase 1. Thus, in Experiments 1 and 2, greater interference from a competing feature on the same source dimension (location) might account for the observed negative effects of item repetition on source memory. To address this possibility, in Experiment 3 we used two different salient source dimensions in Phase 1 (location: four quadrants) and Phase 2 (background colors: four colors). Pictures were presented in one of four quadrants in Phase 1 for a varying number of times, and once in one of the four background colors in Phase 2. We also changed the original background color of each picture (white) to the background color of the presentation screen (gray) to reduce the perception in Phase 1 that each picture was accompanied by a background color.

Previous studies have indicated that individual features are coded separately in an initial stage of visual perception (Treisman \& Schmidt, 1982) and that different visual source dimensions, though both bound to the same items, do not necessarily cue each other (e.g., Starns \& Hicks, 2005; but see Starns \& Hicks, 2008). The suggested independence of different source dimensions at retrieval leads to the prediction that greater item frequency in Phase 1 (associated with greater location information) would not negatively influence source memory for critical source information in Phase 2 (background color). Thus, we hypothesized that if the findings from Experiments 1 and 2 resulted from unequal amounts of location source information across different levels of item frequency, we would not expect to find an effect of item frequency in Experiment 3, where within-dimension source interference was minimized.
Method

\section{Participants}

A group of 37 undergraduate students participated for course credit (mean age: 19.16 [ \pm 1.22$]$; 19 female, 18 male). The data from two participants who correctly guessed the experimental hypothesis and another two who anticipated the subsequent surprise memory test were excluded from the analysis (thus, $N=33$ ).

\section{Design, materials, and procedure}

In Phase 1, item frequency varied (one, four, or 16 presentations), and items were presented in various locations. The stimuli were those used in Experiment 2, with the background changed from white to a uniform gray. Each lowfrequency item was presented once in one quadrant; medium-frequency items were each presented one time in each quadrant; and high-frequency items were each presented four times in each quadrant. Sixteen items were assigned to each frequency condition, and locations were used equally across items within each frequency condition. In Phase 2, object pictures were presented one at a time in the center of the computer screen in one of four background colors (red, green, blue, or yellow). As in Experiments 1 and 2, participants made living/nonliving judgments in Phases 1 and 2. Phase 3 was identical to that of Experiment 2, except that the participants were asked to indicate the Phase 2 background color of each item.

\section{Results and discussion}

A significant effect of item frequency on source memory accuracy was once again obtained, $F(2,64)=9.60, p<.001$, $\eta_{\mathrm{p}}{ }^{2}=.23$ (Fig. 3). Participants' memory for the background

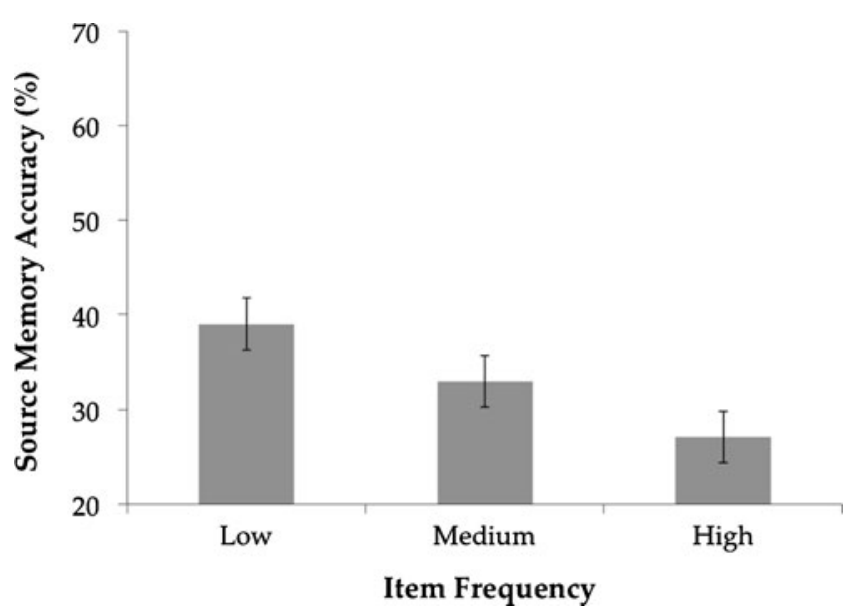

Fig. 3 Source memory accuracy as a function of item frequency in Experiment 3. Error bars are $95 \%$ within-subjects confidence intervals 
color decreased as item frequency increased: The mean accuracies were $39.02 \%, 32.96 \%$, and $27.08 \%$ for the low-, medium-, and high-frequency conditions, respectively, showing a linear trend, $F(1,32)=16.12, p<.001, \eta_{\mathrm{p}}{ }^{2}=.34$.

In Experiment 3, prior item repetition hurt source memory, even though we attempted to minimize differences in the amount of within-dimension source interference across item frequency conditions. Thus, it is unlikely that negative item repetition effects on source memory reflect only source interference at retrieval within the tested source feature dimension. In addition, the results of Experiment 3 provided further evidence against the idea that facilitated item processing from previous item repetition might enhance the binding of item and source information.

\section{Experiment 4}

In Experiments 1 through 3, source information from Phase 2 was acquired incidentally. Intentional learning encourages individuals to focus attention strategically, in a goal-directed fashion, on specific to-be-learned aspects of events - attention that might otherwise be allocated elsewhere under incidentallearning conditions. In Experiment 4, we investigated whether the negative effects of item repetition on source memory observed in Experiments 1 through 3 would hold under intentional-learning instructions that directed participants' deliberate attention to critical source features in Phase 2 through explicit instructions to associate each item with its background color for an upcoming source memory test.

Method

\section{Participants}

A group of 33 undergraduate students (mean age: 18.5 [ \pm 0.88$]$; 14 female, 19 male) participated for course credit.

a

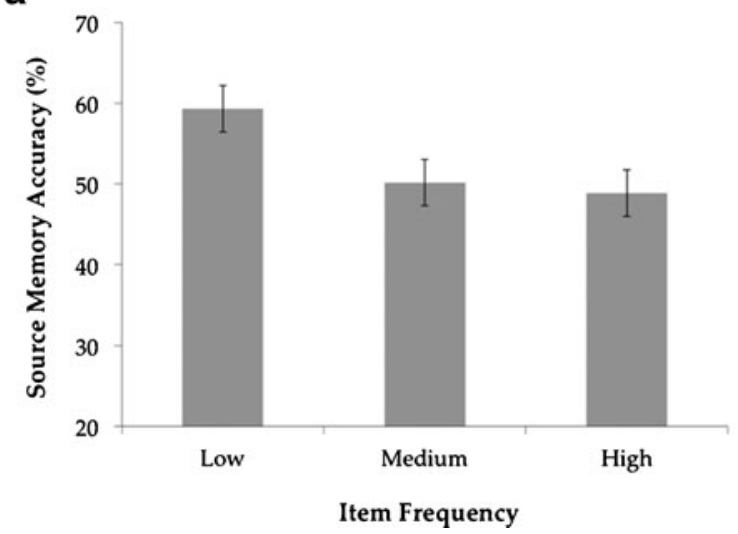

Design, materials, and procedure

The design and stimuli were identical to those of Experiment 3. The procedure was the same as in Experiment 3, except that immediately before Phase 2 , participants were fully informed about the presence and nature of the upcoming source memory test in Phase 3.

Results and discussion

The effect of item frequency on source memory accuracy was significant, $F(2,64)=7.77, p=.001, \eta_{\mathrm{p}}{ }^{2}=.20$ (Fig. $\left.4 \mathrm{a}\right)$. Again, the effect showed a linear trend, $F(1,32)=12.68, p=$ $.001, \eta_{\mathrm{p}}{ }^{2}=.28$. The source memory accuracies for the low-, medium-, and high-frequency conditions were $59.28 \%$, $50.19 \%$, and $48.86 \%$, respectively.

In Experiment 4, we again observed a negative effect of item frequency on source memory, even when participants deliberately attempted to bind critical source information to items. Manipulating deliberate allocation of attention through explicit instructions failed to eliminate the detrimental effects of item frequency on source memory. A discussion of potential, more automatic effects of attentional processing occurring at encoding, in regard to the attentionlikelihood theory and the recursive reminding hypothesis, will follow in the General discussion section.

\section{Experiment 5}

In Experiments 1 through 4, we reliably observed negative effects of item frequency on source memory when participants were exposed to all of the items prior to Phase 2. In Experiment 5, we added to Phase 2 items that had never appeared in Phase 1 ("novel" condition) to assess whether absolute item novelty improves source memory beyond the beneficial effects attributable to relatively low frequency of b

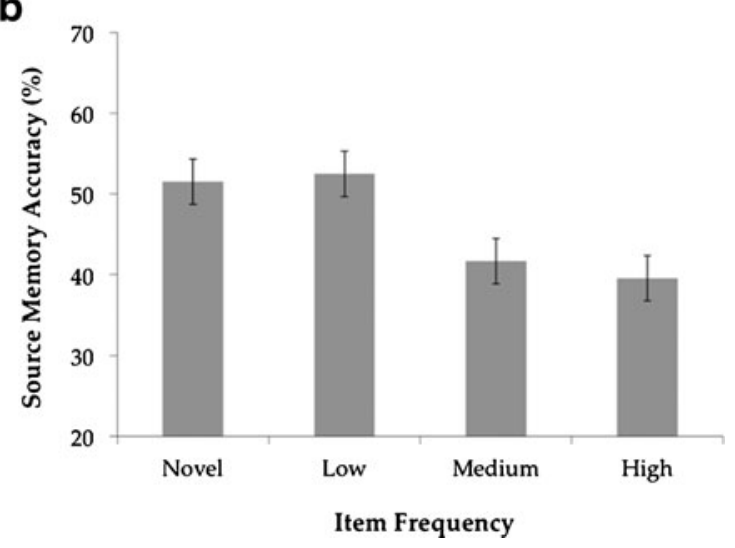

Fig. 4 Source memory accuracy as a function of item frequency in (a) Experiment 4 and (b) Experiment 5. Error bars are 95\% within-subjects confidence intervals 
items. We hypothesized that if absolute item novelty per se determines the efficacy of the episodic encoding of an item and its associated source features (as would be suggested by extending the novelty-encoding hypothesis from item memory to source memory), we should observe better source memory for novel items than for once-presented lowfrequency items.

Method

\section{Participants}

A group of 34 undergraduate students participated for course credit (mean age: 19.21 [ \pm 1.34$] ; 19$ female, 15 male). The data from one participant failed to be properly collected, leaving a final sample of 33 participants.

\section{Design, materials, and procedure}

The experiment involved one within-subjects independent factor (item frequency: novel, low, medium, or high). The stimuli were those used in Experiment 1 with the background color of each drawing changed from white to gray. The procedure was the same as in Experiment 4, except that 16 "novel" pictures were additionally presented only in Phases 2 and 3.

\section{Results and discussion}

The effect of item frequency was again significant, $F(3,96)=$ $11.02, p<.001, \eta_{\mathrm{p}}{ }^{2}=.26$ (Fig. $4 \mathrm{~b}$ ). The mean source memory accuracies were $51.52 \%, 52.46 \%, 41.67 \%$, and $39.58 \%$ for the novel and the low-, medium-, and high-frequency conditions, respectively. For the conditions corresponding to the preceding experiments, the effect of item frequency again showed a linear trend, $F(1,32)=20.39, p<.001, \eta_{\mathrm{p}}{ }^{2}=.28$. Of interest, planned comparisons revealed a nonsignificant difference between the novel and low-frequency conditions, $t(32)=-0.35, p=.73$.

Overall, Experiment 5 replicated the pattern of source memory accuracy found for the corresponding conditions in Experiment 4. Importantly, source accuracy did not significantly differ between the novel and low-frequency items, suggesting that relative item frequency may be more important than absolute novelty in producing effects of item repetition on source memory.

\section{Experiment 6}

In the preceding experiments, we reliably observed negative effects of item repetition on source memory. These experiments did not distinguish between Phase 2, encoding-related effects (i.e., less efficient encoding of source information associated with more frequently presented items) or Phase 3 , retrieval-related differences (i.e., greater interference arising from multiple irrelevant traces at retrieval for more frequently presented items). To provide further evidence regarding the locus of the negative item repetition effect, Experiment 6 reversed the order of Phases 1 and 2. In Experiment 6, items were repeated varying numbers of times subsequent to the encoding of the critical item-source associations. We hypothesized that if the negative effect of item repetition arises at retrieval due to interference from more irrelevant memory traces for items with greater frequency, as suggested by an account emphasizing interference from multiple traces, we should still observe impaired source memory for more frequently presented items. In contrast, if the observed effects result from encoding-related factors, as suggested by theories positing less efficacious encoding of source information for items with more-frequent prior presentations, the negative item repetition effect should no longer be present when, at encoding, the prior frequency for each item is held constant.

\section{Method}

\section{Participants}

A group of 20 undergraduate students participated for pay (\$10) (mean age: 20.5 [ \pm 2.76$] ; 12$ female, 8 male).

\section{Design, materials, and procedure}

The design and stimuli were identical to those of Experiment 2. Relative to Experiments 1 through 5, the order between the item preexposure phase and the itemsource feature association phase was reversed, followed by a third phase testing for source memory for the (now) Phase 1 location information. Otherwise, the procedure was the same as in Experiment 2.

Results and discussion

The mean source memory accuracies were $38.75 \%, 34.06 \%$, and $36.25 \%$ for the low-, medium-, and high-frequency conditions, respectively (Fig. 5). The effect of item frequency was not significant, $F(2,38)=0.82, p=.45$.

When the order of item repetition and source encoding was reversed in Experiment 6, such that source encoding came first, item frequency no longer significantly affected source memory. Though this is a null finding, the absence of a significant effect of item repetition contrasts with the reliable findings in the previous five experiments of a significant effect of item frequency when the repetitions preceded the critical source-encoding phase. Thus, the results 


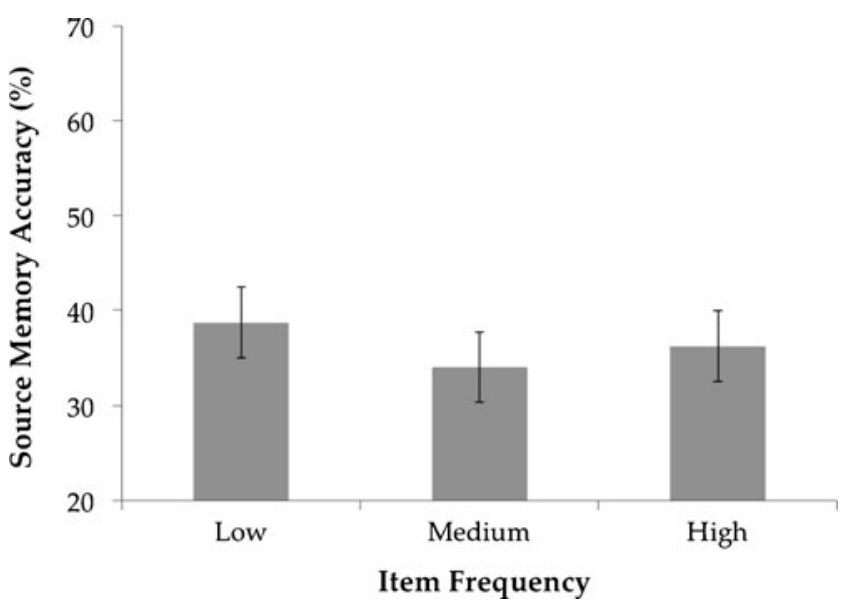

Fig. 5 Source memory accuracy as a function of item frequency in Experiment 6. Error bars are 95\% within-subjects confidence intervals

of Experiment 6 are consistent with the hypothesis of less efficacious source encoding of more frequently presented items.

\section{General discussion}

These experiments demonstrate that (1) prior presentation of an item impairs memory for source-specifying features from a later presentation, and (2) this negative effect of prior item presentation on source memory occurs at source encoding rather than only at retrieval. Source memory accuracy decreased as the number of prior item exposures increased (Exps. 1 and 2). This pattern held when we minimized potential differences in source interference for items of differing frequency by using different source dimensions in the prior item exposure and the critical source-encoding phases (Exp. 3). When deliberate attention was directed to critical source information in Phase 2 with intentionallearning instructions, there was still a source memory disadvantage for medium- and high-frequency items relative to low-frequency items (Exp. 4). When novel items were added in Phase 2, we found better source memory for novel and low-frequency items than for medium- or highfrequency items, but no further benefit for novel as compared to low-frequency items (Exp. 5). Finally, in Experiment 6, when items were repeated subsequent to the encoding of critical source information, item repetition no longer significantly affected source memory, consistent with the hypothesis that the locus of negative item repetition effects observed in the other five experiments was during encoding rather than during the test phase.

Although in Experiment 5 we did not find any advantage for novel over items presented once, there may be circumstances in which novelty would confer a special encoding advantage. Among previous studies showing a recognition memory advantage for novel as compared to familiar items (Äberg \& Nilsson, 2001; Kormi-Nouri et al., 2005; Tulving \& Kroll, 1995), the study most comparable to the present study is that of Äberg and Nilsson, in which they presented items zero, one, two, or three times prior to Phase 2, which involved incidental learning. They found a linear decrease in item recognition memory accuracy as a function of increasing item frequency, including better item memory for novel than for once-presented items. Notably, their familiarization phase involved substantially longer stimulus presentations (7 and $10 \mathrm{~s}$ in Exps. 1 and 2, respectively) than did that of the present study (1 s). Thus, it is likely that the difference in item familiarity between novel and once-presented items was greater in their study than in ours. One potential avenue to pursue in future studies will be to systematically manipulate both the duration and frequency of item presentations and to assess the relative impact of these two factors on the magnitude of the difference in source memory (as well as item memory) between novel versus repeated items. Also, it has been suggested that the recognition advantage for novel items is more pronounced under incidental than under intentional encoding of items in Phase 2 (e.g., Kormi-Nouri et al., 2005). Thus, it is possible that the intentional-learning instructions in Experiment 5 might have diluted a novelty effect.

The fact that we still observed poorer source memory as a function of item frequency under intentional-learning instructions (Exps. 4 and 5) suggests that (a) participants misjudge the amount of strategic, reflective attention required to encode the source features of more frequently presented items, and/or (b) more attention is automatically drawn to lower-frequency items or is automatically drawn away from higher-frequency items. The latter possibility is consistent with an extension to source memory of the theories noted in the introduction that predict less efficacious encoding of more frequently presented items. Both the attention-likelihood theory and the recursive reminding hypothesis propose less attention during encoding of items following more versus fewer prior presentations. However, these theories differ in the specific mechanisms proposed to account for the better memory for less frequent items.

Attention-likelihood theory assumes that different classes of stimuli, such as words that are high versus low in frequency within the English language, evoke differential amounts of attention at encoding, which translates into differences in the number of features that are examined and marked. The attention-likelihood theory suggests that the overall amounts of attention differ across stimulus classes from an early stage of stimulus processing. This is in line with findings that participants allocated longer study time to low-frequency than to high-frequency words when study was self-paced (e.g., Rao \& Proctor, 1984). The recursive reminding hypothesis assumes that the 
phenomenal experience of "being reminded"-occurring in an automatic, involuntary fashion - directs attention to internal memory representations. The number of embedded remindings, which increases as a function of prior item frequency, thus determines how much attention at a given episodic event would be directed internally. Consequently, the amount of attention directed externally to process an item and its associated source features should differ for items with differing prior frequency. The potential negative effect of automatically occurring remindings on encoding of external source features is in line with previous findings of the detrimental effects of generation during encoding on source memory (Jurica \& Shimamura, 1999) and of decreased source memory following focusing on one's own emotional reactions to statements made by two speakers during encoding, as compared with focusing on the speakers' emotions (Johnson, Nolde, \& De Leonardis, 1996). Whereas attention-likelihood theory is not specific about why less-frequent items attract more attention, the recursive reminding hypothesis provides a specific hypothesis about why more-frequent items attract less perceptual attention than do low-frequency items (i.e., they are attracting reflective attention via remindings). Future studies that manipulate the relative demands on external/perceptual versus internal/reflective attention (Chun \& Johnson, 2011) would help clarify the mechanism(s) underlying the negative impact of prior item presentations on source memory.

Finally, it should be noted that in contrast to the present findings, a recent study by Poppenk et al. (2010, Exp. 2) found a prestudy item familiarity advantage in source memory. In their study, intraexperimental item familiarity was manipulated in Phase 1, in which participants were shown some preexperimentally novel foreign proverbs three times each, while making a decision about the origins of each proverb (i.e., South American or Asian). In Phase 2 (i.e., the critical study phase), participants were presented with intraexperimentally familiarized foreign proverbs, preexperimentally familiar English proverbs, and previously unseen preexperimentally novel proverbs and were asked to judge either the vividness or the valence of each proverb. The type of judgment task served as critical source information to be probed in a subsequent surprise source memory test, in which participants had to indicate whether each proverb was judged in regard to its vividness or valence. Poppenk et al. found that regardless of whether item familiarity was preexperimentally or experimentally induced, source memory accuracy was significantly better for familiar than for novel items. Their study differed from the present study in many respects, such as the type of study materials, the type of orienting task, use of the same or of different orienting tasks across Phases 1 and 2, and the type of information serving as a critical source at test (perceptual features vs. records of the cognitive operations engaged). Any of these factors might have contributed to the discrepancy between
Poppenk et al.'s study and the present findings. One interesting possibility is that in the Poppenk et al. study, repeated prestudy of preexperimentally unfamiliar novel foreign proverbs provided participants with multiple opportunities to comprehend unfamiliar proverbs, thereby making them more meaningful, rather than merely increasing their familiarity through repetition (e.g., Bransford \& Johnson, 1972; Chalmers \& Humphreys, 1998). Dissociating the effects of item familiarity and meaningfulness on source memory would help further clarify how specific episodes involving familiar items are encoded and remembered.

In short, the present study reliably found that prior experience with an item impairs memory for subsequently presented source-specifying features. The evidence from these experiments is most consistent with the idea that previous experience with an item reduces the attention directed toward encoding specific details surrounding the item in subsequent presentations. A reasonable interpretation is that when participants are reminded of previous occurrences of an item, reflective attention to these earlier occurrences (i.e., remindings) reduces perceptual attention to source features such as location, color, and so on (Hintzman, 2004). Future efforts exploring conditions yielding advantages and disadvantages of item repetition on memory for the kind of source information that gives rise to the experience of distinct episodic memories should help further explicate the underlying mechanisms by means of which past experiences modulate processes contributing to the formation of new episodic memories.

Author note This research was supported by National Institute on Aging Grant R37AG009253, awarded to M.K.J., and by National Research Foundation of Korea Grant KRF-2011-0003489, awarded to D.-J.Y.

\section{References}

Äberg, C. S., \& Nilsson, L. G. (2001). Facilitation of source discrimination in the novelty effect. Scandinavian Journal of Psychology, 42, 349-357. doi:10.1111/1467-9450.00246

Bransford, J. D., \& Johnson, M. K. (1972). Contextual prerequisites for understanding: Some investigations of comprehension and recall. Journal of Verbal Learning and Verbal Behavior, 11, 717-726. doi:10.1016/S0022-5371(72)80006-9

Chalfonte, B. L., \& Johnson, M. K. (1996). Feature memory and binding in young and older adults. Memory \& Cognition, 24, 403-416. doi:10.3758/BF03200930

Chalmers, K. A., \& Humphreys, M. S. (1998). Role of generalized and episode specific memories in the word frequency effect in recognition. Journal of Experimental Psychology: Learning, Memory, and Cognition, 24, 610-632. doi:10.1037/0278-7393.24.3.610

Chun, M. M., \& Johnson, M. K. (2011). Memory: Enduring traces of perceptual and reflective attention. Neuron, 72, 520-535. doi:10.1016/j.neuron.2011.10.026

Diana, R. A., \& Reder, L. M. (2006). The low-frequency encoding disadvantage: Word frequency affects processing demands. 
Journal of Experimental Psychology: Learning, Memory, and Cognition, 32, 805-815. doi:10.1037/0278-7393.32.4.805

Gardiner, J. M., \& Richardson-Klavehn, A. (2000). Remembering and knowing. In E. Tulving \& F. I. M. Craik (Eds.), The Oxford handbook of memory (pp. 229-244). New York, NY: Oxford University Press.

Gibson, E. J. (1940). A systematic application of the concepts of generalization and differentiation to verbal learning. Psychological Review, 47, 196-229. doi:10.1037/h0060582

Gibson, E. J. (1953). Improvement in perceptual judgments as a function of controlled practice or training. Psychological Bulletin, 50, 401-431. doi:10.1037/h0055517

Glanzer, M., \& Adams, J. K. (1985). The mirror effect in recognition memory. Memory \& Cognition, 13, 8-20. doi:10.3758/ BF03198438

Glanzer, M., \& Adams, J. K. (1990). The mirror effect in recognition memory: Data and theory. Journal of Experimental Psychology: Human Learning and Memory, 2, 21-31. doi:10.1037/02787393.16.1.5

Glanzer, M., Adams, J. K., Iverson, G. J., \& Kim, K. (1993). The regularities of recognition memory. Psychological Review, 100, 546-567. doi:10.1037/0033-295X.100.3.546

Guttentag, R. E., \& Carroll, D. (1997). Recollection-based recognition: Word frequency effects. Journal of Memory and Language, 37, 502-516. doi:10.1006/jmla.1997.2532

Henkel, L. A., Franklin, N., \& Johnson, M. K. (2000). Cross-modal source monitoring confusions between perceived and imagined events. Journal of Experimental Psychology: Learning, Memory, and Cognition, 26, 321-335. doi:10.1037/0278-7393.26.2.321

Hicks, J. L., Marsh, R. L., \& Cook, G. I. (2005). An observation on the role of context variability in free recall. Journal of Experimental Psychology: Learning, Memory, and Cognition, 31, 1160-1164. doi:10.1037/0278-7393.31.5.1160

Hintzman, D. L. (1976). Repetition and memory. In G. H. Bower (Ed.), The psychology of learning and motivation (Vol. 10, pp. 47-91). New York, NY: Academic Press.

Hintzman, D. L. (1988). Judgments of frequency and recognition memory in a multiple-trace memory model. Psychological Review, 95, 528-551. doi:10.1037/0033-295X.95.4.528

Hintzman, D. L. (2004). Judgment of frequency versus recognition confidence: Repetition and recursive reminding. Memory \& Cognition, 32, 336-350. doi:10.3758/BF03196863

Hintzman, D. L. (2010). How does repetition affect memory? Evidence from judgments of recency. Memory \& Cognition, 38, 102-115. doi:10.3758/MC.38.1.102

Hintzman, D. L., \& Waters, R. M. (1970). Recency and frequency as factors in list discrimination. Journal of Verbal Learning and Verbal Behavior, 9, 218-221. doi:10.1016/S0022-5371(70) 80054-8

Holmes, J. B., Waters, H. S., \& Rajaram, S. (1998). The phenomenology of false memories: Episodic content and confidence. Journal of Experimental Psychology: Learning, Memory, and Cognition, 24, 1026-1040. doi:10.1037/0278-7393.24.4.1026

Howell, W. C. (1973). Representation of frequency in memory. Psychological Bulletin, 80, 44-53. doi:10.1037/h0034648

Johnson, M. K., Hashtroudi, S., \& Lindsay, D. S. (1993). Source monitoring. Psychological Bulletin, 114, 3-28. doi:10.1037/ 0033-2909.114.1.3

Johnson, M. K., Nolde, S. F., \& De Leonardis, D. M. (1996). Emotional focus and source monitoring. Journal of Memory and Language, 35, 135-156. doi:10.1006/jmla.1996.0008

Johnson, M. K., \& Raye, C. L. (1981). Reality monitoring. Psychological Review, 88, 67-85. doi:10.1037/0033-295X.88.1.67

Jurica, P. J., \& Shimamura, A. P. (1999). Monitoring item and source information: Evidence for a negative generation effect in source memory. Memory \& Cognition, 27, 648-656. doi:10.3758/ BF03211558

Kelley, C. M., \& Jacoby, L. L. (2000). Recollection and familiarity: Process-dissociation. In E. Tulving \& F. I. M. Craik (Eds.), The Oxford handbook of memory (pp. 215-228). New York, NY: Oxford University Press.

Kirchhoff, B. A., Wagner, A. D., Maril, A., \& Stern, C. E. (2000). Prefrontal-temporal circuitry for episodic encoding and subsequent memory. Journal of Neuroscience, 20, 6173-6180. Retrieved from www.jneurosci.org/content/20/16/6173.full.pdf + html

Kishiyama, M. M., \& Yonelinas, A. P. (2003). Novelty effects on recollection and familiarity in recognition memory. Memory \& Cognition, 31, 1045-1051. doi:10.3758/BF03196125

Kormi-Nouri, R., Nilsson, L. G., \& Ohta, N. (2005). The novelty effect: Support for the novelty-encoding hypothesis. Scandinavian Journal of Psychology, 46, 133-143. doi:10.1111/ j.1467-9450.2005.00443.x

Kumaran, D., \& Maguire, E. A. (2006). An unexpected sequence of events: Mismatch detection in the human hippocampus. PLoS Biology, 4, e424. doi:10.1371/journal.pbio.0040424

Lisman, J. E., \& Grace, A. A. (2005). The hippocampal-VTA loop: Controlling the entry of information into long-term memory. Neuron, 46, 703-713. doi:10.1016/j.neuron.2005.05.002

Loftus, G. R., \& Masson, M. E. J. (1994). Using confidence intervals in within-subject designs. Psychonomic Bulletin \& Review, 1, 476-490. doi:10.3758/BF03210951

Lyle, K. B., \& Johnson, M. K. (2006). Importing perceived features into false memories. Memory, 14, 197-213. doi:10.1080/ 09658210544000060

Marsh, R. L., Cook, G. I., \& Hicks, J. L. (2006). The effect of context variability on source memory. Memory \& Cognition, 34, 15781586. doi:10.3758/BF03195921

Miozzo, M., \& Caramazza, A. (2003). When more is less: A counterintuitive effect of distractor frequency in the picture-word interference paradigm. Journal of Experimental Psychology. General, 132, 228-252. doi:10.1037/0096-3445.132.2.228

Naveh-Benjamin, M., Craik, F. I. M., Guez, J., \& Dori, H. (1998). Effects of divided attention on encoding and retrieval processes in human memory: Further support for an asymmetry. Journal of Experimental Psychology: Learning, Memory, and Cognition, 24, 1091-1104. doi:10.1037/0278-7393.24.5.1091

Naveh-Benjamin, M., \& Guez, J. (2000). Effects of divided attention on encoding and retrieval processes: Assessment of attentional costs and a componential analysis. Journal of Experimental Psychology: Learning, Memory, and Cognition, 26, 1461-1482. doi:10.1037/0278-7393.26.6.1461

Newby, R. W. (1981). Contextual cues in multiple-item recognition. The American Journal of Psychology, 94, 513-526. doi:10.2307/ 1422260

Overton, R. C., \& Adolphson, C. J. (1979). Multiple trace retrieval: A trace-to-trace retrieval process? Journal of Experimental Psychology: Human Learning and Memory, 5, 485-495. doi:10.1037/0278-7393.5.5.485

Poppenk, J., Köhler, S., \& Moscovitch, M. (2010). Revisiting the novelty effect: When familiarity, not novelty, enhances memory. Journal of Experimental Psychology: Learning, Memory, and Cognition, 36, 1321-1330. doi:10.1037/a0019900

Postman, L. (1971). Transfer, interference, and forgetting. In J. W. Kling \& L. A. Riggs (Eds.), Woodworth and Schlosberg's experimental psychology (3rd ed., pp. 1019-1132). New York, NY: Holt, Rinehart \& Winston.

Rao, K. V., \& Proctor, R. W. (1984). Study-phase processing and the word frequency effect in recognition memory. Journal of Experimental Psychology: Learning, Memory, and Cognition, 10, 386-394. doi:10.1037/0278-7393.10.3.386 
Roediger, H. L., \& McDermott, K. B. (1993). Implicit memory in normal human subjects. In F. Boller \& J. Grafman (Eds.), Handbook of neuropsychology (Vol. 8, pp. 63-131). Amsterdam, TheNetherlands: Elsevier.

Rugg, M. D., Cox, C. J. C., Doyle, M. C., \& Wells, T. (1995). Eventrelated potentials and the recollection of low and high frequency words. Neuropsychologia, 33, 471-484. doi:10.1016/0028-3932 (94)00132-9

Snodgrass, J. G., \& Vanderwart, M. (1980). A standardized set of 260 pictures: Norms for name agreement, image agreement, familiarity, and visual complexity. Journal of Experimental Psychology: Human Learning and Memory, 6, 174-215. doi:10.1037/02787393.6.2.174

Starns, J. J., \& Hicks, J. L. (2005). Source dimensions are retrieved independently in multidimensional monitoring tasks. Journal of Experimental Psychology: Learning, Memory, and Cognition, 31, 1213-1220. doi:10.1037/0278-7393.31.6.1213

Starns, J. J., \& Hicks, J. L. (2008). Context attributes in memory are bound to item information, but not to one another. Psychonomic Bulletin \& Review, 15, 309-314. doi:10.3758/PBR.15.2.309
Treisman, A., \& Schmidt, H. (1982). Illusory conjunctions in the perception of objects. Cognitive Psychology, 14, 107-141. doi:10.1016/0010-0285(82)90006-8

Tulving, E. (1983). Elements of episodic memory. New York, NY: Oxford University Press.

Tulving, E., \& Kroll, N. (1995). Novelty assessment in the brain and long-term memory encoding. Psychonomic Bulletin \& Review, 2, 387-390. doi:10.3758/BF03210977

Tulving, E., Markowitsch, H. J., Craik, F. I. M., Habib, R., \& Houle, S. (1996). Novelty and familiarity activations in PET studies of memory encoding and retrieval. Cerebral Cortex, 6, 71-79. doi:10.1093/cercor/6.1.71

Tulving, E., \& Schacter, D. L. (1990). Priming and human memory systems. Science, 247, 301-306. doi:10.1126/science.2296719

Underwood, B. J. (1949). Proactive inhibition as a function of time and degree of prior learning. Journal of Experimental Psychology, 39, 24-34. doi: $10.1037 / \mathrm{h} 0059550$

Wixted, J. T., \& Stretch, V. (2004). In defense of the signal detection interpretation of remember/know judgments. Psychonomic Bulletin \& Review, 11, 616-641. doi:10.3758/BF03196616 